\title{
INFLUENCIA DE LA PARTICIPACIÓN SOCIAL EN EL PROCESO DE REHABILITACIÓN BASADA EN COMUNIDAD, EN LAS PERSONAS CON DISCAPACIDAD DEL MUNICIPIO DE PAMPLONA - NORTE DE SANTANDER
}

\author{
SOCIAL PARTICIPATION INFLUENCE IN PROCESS BASED ON COMMUNITY \\ REHABILITATION, ON PERSONS WITH DISABILITIES OF THE CITY OF PAMPLONA - \\ NORTE DE SANTANDER
}

\section{Magda Milena Contreras Jaureguí ${ }^{1}$, Yenni Omaira Celis Romero², Javier Martinez ${ }^{3}$, Adriana Margarita Gutiérrez Ávila y Katherine Ortiz Ruiz ${ }^{4}$}

\begin{abstract}
Resumen
La Organización Mundial de la Salud en el 2004, define la Rehabilitación Basada en Comunidad como: "Una estrategia de desarrollo comunitario para la Rehabilitación, la igualdad de oportunidades y la integración social de todas las personas con discapacidad, esta se aplica gracias al esfuerzo conjunto de las propias personas con discapacidad, sus familias, organizaciones, comunidades, servicios gubernamentales y no gubernamentales en salud, educación, trabajo, social y otros". Que permite que las personas con discapacidad cuenten con la Participación Social, la cual se define como un grupo organizado, activo, comprometido y corresponsable de los actores de la sociedad para alcanzar beneficios comunes.

Metodología: Es una investigación tipo descriptivo no experimental- transaccional, con diseño mixto, técnicas de recolección de la información de tipo cuantitativo y cualitativo (cartografía social, diarios de campo y entrevistas a profundidad, encuestas, de las cuales se obtienen información de tipo social, pedagógico y clínico.
\end{abstract}

\section{Palabras Claves:}

Ocupación humana, personas con discapacidad, rehabilitación basada en comunidad.

\begin{abstract}
The World Health Organization in 2004, defines Community Based Rehabilitation as "A strategy of community development for the rehabilitation, equalization of opportunities and social integration of all people with disabilities, this is applied through the joint efforts of the persons with disabilities, their families, organizations, communities, government and non-government services in health, education , labor, social and others. "Which allows people with disabilities have the Social Participation, which is defined as an organized , active, committed and co- actors of society group to achieve common benefits.

Methodology: A descriptive research experimentally not transactional, with mixed design, technical information collection of quantitative and qualitative (social mapping, field notes and in-depth interviews, surveys, from which information is obtained from social type, educational and clinical.
\end{abstract}

\section{Keywords:}

Human occupation, people with disabilities, community -based rehabilitation.

1 Terapeuta Ocupacional. Profesora asistente Universidad de Pamplona. Magister en Riesgo Psicosocial. dto@unipamplona.edu.co

2 Terapeuta Ocupacional. Profesora Universidad de Pamplona. Especialista en Educación Especial e inclusión social. yoldcm@hotmail.com

3 Fisioterapeuta. Universidad de Pamplona. Epid_javier@hotmail.com

4 Estudiantes Universidad de pamplona Kathortiz-@hotmail.com; Adrym831-@hotmail.com 


\section{INTRODUCCIÓN}

La Organización Mundial de la Salud en el 2004, y el Ministerio de asuntos sociales y salud, , 2003. Helsinki, Finlandia. define la Rehabilitación Basada en Comunidad como: "Una estrategia de desarrollo comunitario para la Rehabilitación, la igualdad de oportunidades y la integración social de todas las personas con discapacidad, esta se aplica gracias al esfuerzo conjunto de las propias personas con discapacidad, sus familias, organizaciones, comunidades, servicios gubernamentales y no gubernamentales en salud, educación, trabajo, social y otros". Que permite que las personas con discapacidad cuenten con la Participación Social, la cual se define como un grupo organizado, activo, comprometido y corresponsable de los actores de la sociedad para alcanzar beneficios comunes.

Según la OMS en el 2012, Guía para la Rehabilitación Basada en la Comunidad, según Lord JE, Guernsey KN, Balfe JM, Karr VL, Flowers N (eds), define La transversalidad dentro del ámbito comunitario como el ende que organiza e implementa a través de la estrategia de rehabilitación basada en comunidad (RBC), las acciones centradas en las personas con discapacidad, sus familias y la comunidad, ordenadas con base en los fundamentos de la RBC, la estrategia promocional de calidad de vida y los conceptos que fundamentan el desarrollo comunitario, articuladas con los principios que rescatan la promoción, el desarrollo y la restitución de los derechos y la autonomía de las personas y los colectivos.

Desde esta articulación, se busca ofrecer respuestas a las necesidades de participación e inclusión de la población con discapacidad en consonancia con el objetivo del ámbito, "Fortalecer el tejido social para mejorar la calidad de vida y la ciudadanía en salud, a través de la movilización social, la gestión de la salud, el fortalecimiento de habilidades y competencias sociales y el reconocimiento, desarrollo y sostenibilidad de redes primarias y secundarias (grupos y organizaciones sociales)" Ley General de Desarrollo Social (Nueva Ley DOF 20-01-2004).

Esta acción hace parte de la categoría "procesos para la restitución de la autonomía de los sujetos individuales y colectivos en el territorio", e integra el desarrollo y fortalecimiento de habilidades y competencias comunitarias y dentro de la estrategia de rehabilitación basada en comunidad responde al eje de formación. Está dirigida a cuidadores/as de personas con discapacidad, se considera el espacio de apoyo y tiempo de respiro que se ofrece a estas personas para la realización de actividades complementarias de crecimiento y bienestar personal, autocuidado, esparcimiento y manejo asistencial en el hogar desde una perspectiva en terapia ocupacional. (Ministerio de salud y protección social 2012 p 4)

Según Blanco y Rodriguez (2012; .p.11 -12), en este sentido, contempla actividades de tipo formativo, educativo, de autoconocimiento, integración social, de desarrollo de habilidades básicas, creativas, expresivas, de comunicación, recreativas y socio-culturales que permitan fortalecer su autoestima y autoconcepto, manejar adecuadamente su tiempo libre, implementar y desarrollar estrategias de Autocuidado, adquirir destrezas en el manejo y cuidado de las personas con discapacidad y tener la posibilidad de compartir con otros sentimientos relacionados con la responsabilidad del cuidado de las personas con discapacidad.

Siendo fundamental para el desarrollo sistemático de la democracia, a partir de un grupo social u organización cuyos integrantes favorecen la participación conjunta de actores diversos que colaboran en la construcción de una situación deseada, resolviendo asuntos que beneficien a la sociedad, afirmando de este modo que existe la participación social. Es un papel primordial del Terapeuta Ocupacional en los procesos de RBC dar a conocer la estrategia, Realización de intervenciones asistenciales, en la preparación de la participación, convertirse en sus propios defensores, favorecer la implementación del programa, estableciendo el desarroIlo del mismo, tanto en el gobierno como en la comunidad, convirtiéndose en el núcleo o eje central del ejercicio profesional y de su abordaje desde el concepto de ocupación humana centrándose en la actividad y participación en la vida diaria, el enfoque basado en el problema de las ocupaciones diarias, la práctica en Rehabilitación, haciendo hincapié en la creatividad para adaptar la solución de problemas a las necesidades de la persona. (OMS 2007.p.172).

\section{MATERIALES Y MÉTODOS}

\section{Diseño de Investigación}

Taylor y Bodgan (1986. p 15) consideran que el diseño de investigación "es el plan o estrategia para recolectar y analizar información con el fin de llegar a 
conclusiones basadas en evidencia sólida y no en un razonamiento defectuoso o en meras opiniones".

Según lo anterior descrito la investigación en su desarrollo utilizo el diseño de investigación:

Mixto. Hernández, Sampieri y Mendoza (2008): P 546 enmarcan el enfoque mixto de la investigación en un proceso que recolecta, analiza y vinculación de datos cuantitativos y cualitativos en un mismo estudio o una serie de investigaciones para responder a un planteamiento del problema. Por lo cual, se usan métodos de los enfoques cuantitativos y cualitativos e intervienen datos cuantitativos y cualitativos y viceversa.

\section{Tipos de Investigación}

Investigación no experimental Mertens (2005. p 184) "Es aquella que se realiza sin manipular deliberadamente variables. Es decir, donde no hacemos variar intencionalmente las variables independientes. Lo que hacemos en la investigación no experimental es observar fenómenos tal y como se dan en su contexto natural, para después analizarlos".

Se tiene en cuenta este tipo de investigación puesto que la población a abordar no requiere ser expuesta ante una situación o evento en particular para promover la discapacidad de las personas, por el contrario se requiere de un proceso observacional, bien sea llegando hasta la localidad la cual habitan en este caso en sus lugares de residencias sin provocar o influir en la discapacidad puesto que esta ya existe y no permite ser manipulada.

\section{Población y Muestra}

\section{Población}

Selltiz, et al. (1980). Es el conjunto de todos los casos que concuerdan con una serie de especificaciones, sobre la cual se pretenden generalizar los resultados. La población total o universal hace referencia a 249 personas con discapacidad vinculadas a la Alcaldía de Pamplona Norte de Santander y asociaciones inscritas a la misma. Liderado por la Secretaria de Salud a cargo de la Dr. Martha Maldonado.

\section{Muestra selectiva}

Hernández, S. (2008). La define como una muestra deliberadamente elegida utilizando un plan de muestreo que permite desestimar la presencia de ciertos materiales que presenten ciertas características y/o seleccionar sólo materiales con otras características relevantes. Se toma una población muestra de 100 personas con discapacidad, en edades comprendidas de $0-18$ años. Muestra que se disminuyó a 60 al momento de la disponibilidad de tiempo, situación de las familias y el deseo de participar en la investigación.

\section{Instrumentos}

Los instrumentos que se utilizaron para esta investigación fueron Instrumento de Valoración Inicial, Consentimiento Informado, Visita Fallida y Lista de Chequeo.

Instrumento de Valoración Inicial. Engloba la dimensión de "Factores Contextuales" contemplando ambientales (físico, social, actitudinales en el cual la gente vive y dirige sus vidas) y los personales deben ser codificados desde la perspectiva de la persona cuya situación está siendo y la describe.

Consentimiento informado, siendo este diligenciado por la madre o el cuidador, debido a que estos son menores de edad que presentan una situación de discapacidad. Este consentimiento es un documento en el cual se establece y da conocimiento de la investigación para que así autoricen su participación en ella obtener información sobre las personas con discapacidad y el entorno en el cual participa; ser fotografiadas, grabadas o videos.

Lista de Chequeo. Es un formulario que nos permite determinar la participación de las personas con discapacidad, en relación a tres entornos; social, físico y cultural. Se entrevista al cuidador o persona con discapacidad en forma verbal, estas respuestas son grabadas por una de las Terapeutas Ocupacionales y la otra es diligenciando el formato el cual permite señalar con una X la calificación presente o ausente. Cuenta con 17 preguntas distribuidas en los 3 contextos anteriormente mencionados, así: El entorno social; 7 preguntas, físico; 5 y finalmente el cultural; 5 interrogantes. Estas respuestas posteriormente serán tabuladas teniendo en cuenta lo diligenciado en el formato y así determinar el nivel de participación de la persona con discapacidad en los diferentes entornos o contextos.

Visita fallida. Es un formato el cual se diligencia cuando al llegar al domicilio de las personas con dis- 
capacidad no se logra vincular a la investigación por diversos motivos referenciados por los mismos, en este se incluye los datos de la persona con discapacidad o cuidador, edad del mismo, barrio, fecha, hora de visita y las observaciones las cuales especifican el motivo de la no vinculación al proyecto.

\section{Confiabilidad y validez de los instrumentos}

Instrumento de valoración inicial. Tomado de: Carmén Elisa Puyana M.D. El Familiograma, Dpto. Salud Familiar. Universidad del Valle, Santiago de Cali, 1989 y se soporta de la clasificación de funcionamiento, discapacidad y salud 2001 de la OMS.

La Lista de chequeo; Sometida a juicio de expertos ya que este ofrece una opinión informada de personas con trayectoria en el tema, reconocidas por otros como expertos cualificados en éste, y que pueden dar información, evidencia, juicios y valoraciones., además los expertos pueden estar relacionados por educación similar, entrenamiento, experiencia, entre otros;. esta lista de chequeo fue evaluada por dos terapeutas ocupacionales expertos en el área, enviando con anterioridad el documento a los profesionales expertos y conocedores del tema, quienes revisaron la lista de chequeo, que tiene el fin determinar el nivel de participación de las personas en condición de discapacidad.

\section{Resultados}

Según la cartografía social empleada dentro del proyecto de investigación de Rehabilitación Basada en la Comunidad para las personas con Discapacidad, se muestra cada sector abordado por las Terapeutas Ocupacionales a cargo del mismo, encontrando aumento de la población con Discapacidad, a nivel del sector centro, seguido de este; Camellón, Santa Marta, Cristo Rey, Juan XXIII, San Ignacio, San francisco, Brighton, Zulia, San pedro y Santa Cruz, estos fueron abarcados de esta manera según las caracterizaciones realizadas a diario, además agrupándose territorios cercanos para proporcionar información donde se conozca la disposición de elementos físicos y artificiales, con relación a la superficie terrestre de cada barrio y posteriormente realizándose la representación en un espacio reducido con información espacial de diversas escalas, con el objetivo de disponerse al alcance de las miradas de cada uno de los espectadores, proporcionando datos y aspectos de un territorio que no podrían ser alcanzados al menos que este sea recorrido. Por otra parte la cartografía es el resultado fundamental de un procedimiento mediante el cual representa un espacio tomado en consideración aspectos que son relevantes para su conocimiento y transformación, partiendo desde la esquematización significativa de un territorio o espacio determinado, posibilitando el manejo sistemático de la información que se desea resaltar, conocimientos del mismo, datos específicos de las familias, personas con Discapacidad y sus comunidades, haciendo referencia dentro de cada tabla el género, institucionalización, rehabilitación, rango de edad de la personas abarcadas y de sus cuidadores, obteniendo de esta manera una búsqueda dinámica, territorios marginados, flujos e interacciones encontradas dentro del municipio de Pamplona - Norte de Santander.

Desde el campo de Terapia Ocupacional, la cartografía social se toma durante el proceso investigativo como una herramienta de tipo metodológico, para así ayudar al análisis de cada entorno abarcado dentro de la Ciudad, así mismo tener un acercamiento de la población con Discapacidad frente a un espacio geográfico, socioeconómico e histórico-cultural. Por otro lado el ejercicio de esta técnica facilita la construcción de un conocimiento de forma colectiva, logando la elaboración de los diferentes mapas anexados por territorios, el cual cada uno enfatiza los métodos de comunicación entre los participantes y colocándose en evidencia los diferentes tipos de saberes y poder llegar a una imagen colectiva de cada sector enfocado. Por otra parte nos permite obtener consciencia de las realidades existentes dentro de los barrios, además de los conflictos y las habilidades individuales y colectivas de cada persona, abriendo de manera caminos hacia una reflexión compartida para consolidar lecturas previas y visiones frente a los espacios recorridos y tiempos específicos, invitando de esta forma a la reflexión y a la acción consciente para el beneficio común.

Partiendo estas necesidades expuestas dentro de los mapas, se elabora una serie de actividades de rehabilitación integral, enfocadas desde el área de Terapia Ocupacional para las personas con discapacidad, sus familias y comunidades, partiendo de la disminución de estos mismos frente a la participación social en programas dirigidos en cuanto cultura, deportes, religioso o juvenil, entre otras, lo cual permite dar mayor dedicación frente al proceso de la Rehabilitación Basada en la Comunidad y contribuir 
al crecimiento personal de los niños, niñas y adolescentes con Discapacidad, mediante el desarrollo de rehabilitación, vinculando activamente a su familia, comunidad, entidades gubernamentales y no gubernamentales que trabajan en beneficio de estas personas, promoviendo su calidad de vida a partir de la restricción de sus derechos.
La población beneficiaria de Pamplona - Norte de Santander, corresponde a niños, niñas y adolescentes habitantes del sector urbano, a quienes se les realizo el proceso de caracterización y la vinculación propia de sus familias como sujetos activos dentro del proceso de la Rehabilitación Basada en la Comunidad, formativo para la satisfacción y logros de los objetivos planteados dentro de la investigación.

Tabla 1.

Distribución de las varaibles sociodemográficas por barrio.

\begin{tabular}{|c|c|c|c|c|c|c|c|c|c|c|c|c|c|}
\hline & 冚 & $\begin{array}{l}\frac{5}{0} \\
\frac{0}{0} \\
\frac{.00}{0} \\
0\end{array}$ & 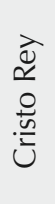 & $\frac{\sqrt{0}}{\overline{\bar{\Xi}}}$ & $\frac{.0}{\bar{N}}$ & 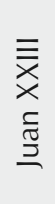 & 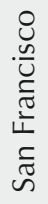 & 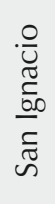 & 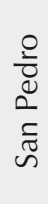 & 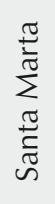 & 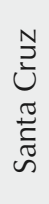 & 胥 & $\begin{array}{l}\frac{0}{\pi} \\
\frac{\pi}{0} \\
0 \\
0 \\
0\end{array}$ \\
\hline & $\mathrm{N}$ & $\mathrm{n}$ & $\mathrm{n}$ & $\mathrm{n}$ & $\mathrm{n}$ & $\mathrm{n}$ & $\mathrm{n}$ & $\mathrm{n}$ & $\mathrm{n}$ & $\mathrm{N}$ & $\mathrm{n}$ & $\mathrm{n}$ & $\%$ \\
\hline Total & 13 & 2 & 7 & 9 & 2 & 6 & 5 & 5 & 2 & 8 & 1 & 60 & $100 \%$ \\
\hline \multicolumn{14}{|l|}{ Género } \\
\hline Femenino & 7 & 1 & 2 & 6 & 1 & 2 & 2 & 5 & 0 & 5 & 1 & 32 & $53,3 \%$ \\
\hline Masculino & 6 & 1 & 5 & 3 & 1 & 4 & 3 & 0 & 2 & 3 & 0 & 28 & $46,7 \%$ \\
\hline \multicolumn{14}{|l|}{ Rehabilitación } \\
\hline Sí & 10 & 0 & 4 & 5 & 1 & 2 & 2 & 2 & 1 & 5 & 1 & 33 & $55,0 \%$ \\
\hline $\mathrm{No}$ & 3 & 2 & 3 & 4 & 1 & 4 & 3 & 3 & 1 & 3 & 0 & 27 & $45,0 \%$ \\
\hline \multicolumn{14}{|l|}{ Institucionalizado } \\
\hline Sí & 8 & 0 & 5 & 5 & 2 & 4 & 2 & 3 & 1 & 6 & 1 & 37 & $61,6 \%$ \\
\hline No & 5 & 2 & 2 & 4 & 0 & 2 & 3 & 2 & 1 & 2 & 0 & 23 & $38,4 \%$ \\
\hline \multicolumn{14}{|l|}{ Seguridad Social } \\
\hline Sí & 13 & 2 & 7 & 9 & 2 & 6 & 5 & 5 & 2 & 8 & 1 & 60 & $100 \%$ \\
\hline $\mathrm{No}$ & 0 & 0 & 0 & 0 & 0 & 0 & 0 & 0 & 0 & 0 & 0 & 0 & $0 \%$ \\
\hline \multicolumn{14}{|c|}{ Edad de persona con discapacidad } \\
\hline $0-3$ años & 0 & 0 & 0 & 0 & 0 & 0 & 0 & 0 & 0 & 0 & 0 & 0 & $0,0 \%$ \\
\hline 3-6 años & 1 & 0 & 1 & 0 & 1 & 1 & 0 & 0 & 0 & 2 & 0 & 6 & $10,0 \%$ \\
\hline 6-9 años & 3 & 0 & 2 & 3 & 1 & 0 & 1 & 2 & 0 & 5 & 0 & 17 & $28,3 \%$ \\
\hline 9-12 años & 1 & 0 & 0 & 0 & 0 & 1 & 1 & 1 & 0 & 0 & 0 & 4 & $6,7 \%$ \\
\hline $12-15$ años & 2 & 0 & 1 & 1 & 0 & 1 & 1 & 0 & 0 & 1 & 0 & 7 & $11,7 \%$ \\
\hline 15 a 18 años & 6 & 2 & 3 & 5 & 0 & 3 & 2 & 2 & 2 & 0 & 1 & 26 & $43,3 \%$ \\
\hline \multicolumn{14}{|l|}{ Edad del cuidador } \\
\hline 25-35 años & 1 & 0 & 3 & 1 & 2 & 1 & 0 & 0 & 0 & 4 & 0 & 12 & $20,0 \%$ \\
\hline 35-45 años & 5 & 1 & 2 & 4 & 0 & 2 & 4 & 0 & 0 & 3 & 0 & 21 & $35,0 \%$ \\
\hline 45-55 años & 5 & 1 & 1 & 4 & 0 & 2 & 0 & 5 & 2 & 1 & 1 & 22 & $36,7 \%$ \\
\hline 55-65 años & 2 & 0 & 0 & 0 & 0 & 1 & 1 & 0 & 0 & 0 & 0 & 4 & $6,7 \%$ \\
\hline 65-75 años & 0 & 0 & 1 & 0 & 0 & 0 & 0 & 0 & 0 & 0 & 0 & 1 & $1,7 \%$ \\
\hline $\begin{array}{c}\text { Personas que requieren } \\
\text { ayuda técnica }\end{array}$ & 2 & 1 & 4 & 1 & 1 & 2 & 2 & 1 & 0 & 2 & 1 & 17 & $100 \%$ \\
\hline
\end{tabular}


Figura 1.

Cartografía por sectores de la ciudad de Pamplona.

\begin{tabular}{|l|l|}
\hline SECTOR CENTRO Sector Brighton & \\
\hline & \\
\hline Sector Cristo Rey & Sector Camellón \\
\hline Sector Zulia & Sector San Ignacio \\
\hline
\end{tabular}




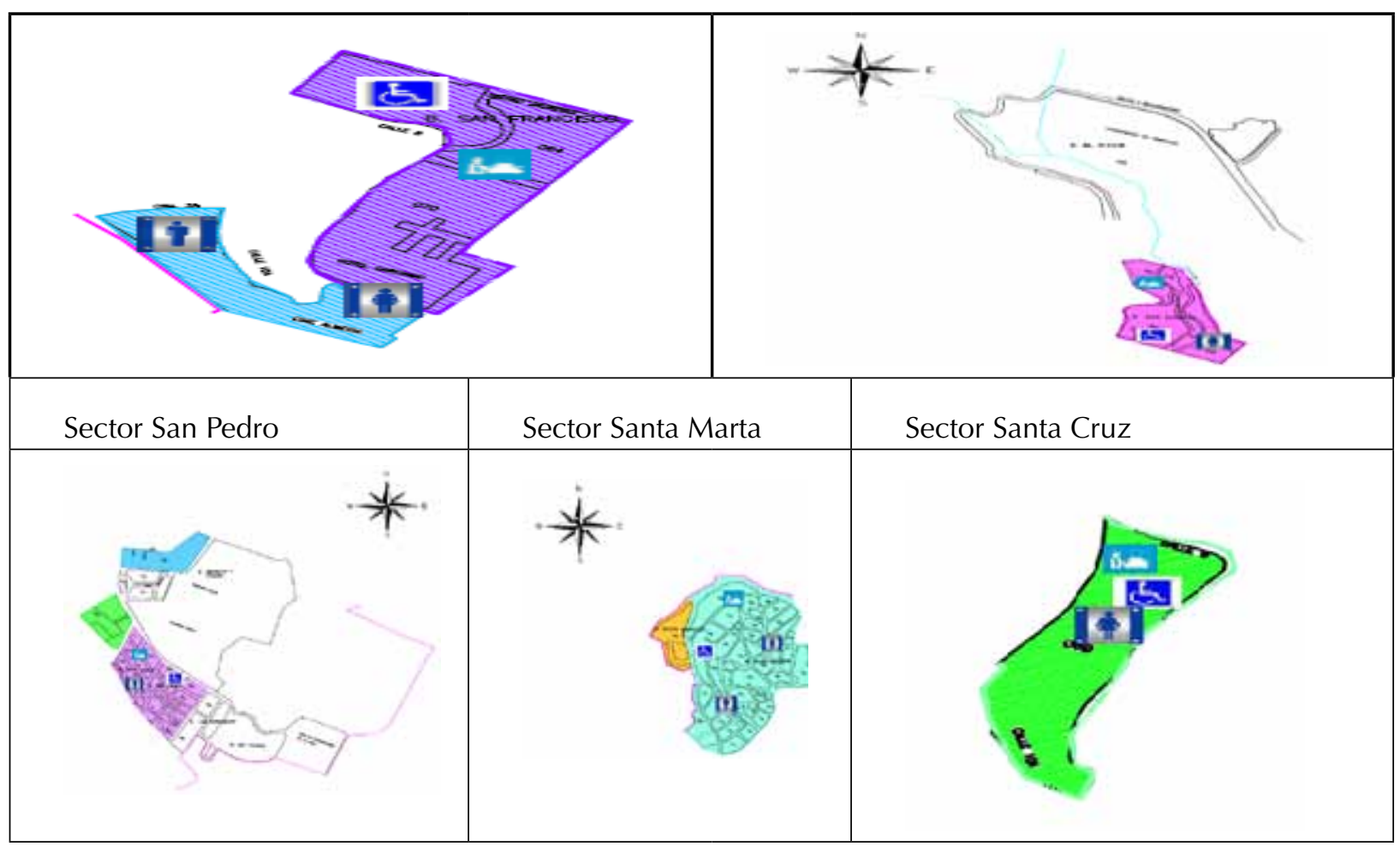

Tabla 2. Distribución por entorno social, físico y social

\begin{tabular}{|c|c|c|c|c|}
\hline Entorno social & \multicolumn{2}{|c|}{ Presente } & \multicolumn{2}{c|}{ Ausente } \\
\hline $\begin{array}{c}\text { Recibe usted buen trato por parte de los miembros de su familia. } \\
\text { tran institucionalizadas. }\end{array}$ & 60 & $100 \%$ & 0 & $0,0 \%$ \\
\hline $\begin{array}{c}\text { Existe algún tipo de discriminación frente a los menores en condición de discapacidad. } \\
\text { Dentro de la comunidad a la cual pertenece, las Personas Con Discapacidad se encuen- }\end{array}$ & 42 & $70,0 \%$ & 18 & $30,0 \%$ \\
\hline $\begin{array}{c}\text { Las personas en condición de discapacidad que usted conoce, hacen parte de organiza- } \\
\text { ciones, grupos sociales establecidos o asociaciones. }\end{array}$ & 24 & $73,3 \%$ & 16 & $26,7 \%$ \\
\hline $\begin{array}{c}\text { Considera que el contexto familiar al que pertenece la Persona Con Discapacidad brin- } \\
\text { da motivadores o existe unión familiar. }\end{array}$ & 60 & $100 \%$ & 0 & $0,0 \%$ \\
\hline $\begin{array}{c}\text { Las Personas Con Discapacidad tienen conocimiento de los programas de Rehabilita- } \\
\text { ción. }\end{array}$ & 42 & $70,0 \%$ & 18 & $30,0 \%$ \\
\hline El gobierno cuenta con políticas de Discapacidad. & 24 & $40,0 \%$ & 36 & $60,0 \%$ \\
\hline Entorno Fisico & & & & \\
\hline La Persona Con Discapacidad cuenta con algún dispositivo de asistencia. & 19 & $31,7 \%$ & 41 & $68,3 \%$ \\
\hline El hogar en el que reside posee barreras que dificulta su movilidad. & 27 & $45,0 \%$ & 33 & $55,0 \%$ \\
\hline Pertenece a algún programa dirigido a las Personas Con Discapacidad (Recreación, \\
Deporte, Religioso o Juvenil). & 12 & $20,0 \%$ & 48 & $80,0 \%$ \\
\hline El contexto en el que se desarrolla ofrece condiciones de accesibilidad. & 25 & $41,7 \%$ & 35 & $58,3 \%$ \\
\hline
\end{tabular}




\begin{tabular}{|c|c|c|c|c|}
\hline \multirow[b]{2}{*}{ Cree que existen limitantes al acceder a los servicios de salud (terreno geográfico). } & \multicolumn{2}{|c|}{ Presente } & \multicolumn{2}{|c|}{ Ausente } \\
\hline & 47 & $78,3 \%$ & 13 & $21,7 \%$ \\
\hline \multicolumn{5}{|l|}{ Entorno Cultural } \\
\hline El entorno familiar permite espacios para la expresión de sus ideas o pensamientos. & 59 & $98,3 \%$ & 1 & $1,7 \%$ \\
\hline El hogar donde reside es propicio para la construcción y desarrollo de valores. & 60 & $100 \%$ & 0 & $0,0 \%$ \\
\hline $\begin{array}{l}\text { Recibe aportes económicos por entes gubernamentales o no gubernamentales para } \\
\text { beneficios de la Personas Con Discapacidad. }\end{array}$ & 12 & $20,0 \%$ & 48 & $80,0 \%$ \\
\hline $\begin{array}{c}\text { Considera que la presencia de determinada discapacidad, afecta el desempeño de los } \\
\text { roles de la Personas Con Discapacidad. }\end{array}$ & 50 & $83,3 \%$ & 10 & $16,7 \%$ \\
\hline Tiene conocimiento sobre los derechos de las personas con discapacidad. & 27 & $45,0 \%$ & 33 & $55,0 \%$ \\
\hline
\end{tabular}

\section{DISCUSIÓN}

Los resultados que se mostraron dentro de cada una de la cartografía realizada en el municipio de Pamplona/Norte de Santander - Colombia, se evidencia mayor cantidad de población con Discapacidad en el sector del Centro, aumento de discapacidades en el género femenino con un porcentaje de $52 \%$ y $48 \%$ en masculino, a nivel de rehabilitación se encontró que el 52.5\% asiste a los procesos de recuperación y el $47.5 \%$; no se vinculan con ninguno de ellos. También se logra identificar los rangos de edades de las personas con Discapacidad, tornándose un resultado del $46 \%$ de los caracterizados, con edades comprendidas entre los 15 a 18 años de edad (adolescencia), afectando el desempeño de los roles, como lo es el escolar y por ende su proceso vocacional, teniendo en cuenta sus intereses, que pueden verse afectados y así mismo ocasionan dificultades en la formación de su identidad y personalidad; además de los problemas académicos. Con relación a la edad de los familiares o cuidadores presenta una equivalencia del $42 \%$, con rangos entre 45 a 55 años, ubicándose en una etapa de madurez y adultez donde esta población muestra poca motivación por dar cumplimiento a sus propias necesidades o expectativas, ya que estos; en la gran mayoría de los casos tienen como prioridad las necesidades y expectativas de vida de las personas a quienes están a su cargo.

Frente a la interpretación de la Lista de Chequeo, se logra identificar en el Entorno Físico que 48 familias de las 60 personas con Discapacidad, responden que los menores no pertenecen a ningún programa dirigido para los mismo, con relación a Deportes, Religioso, Juvenil u otro, teniendo en cuenta la dificultad existente en cuanto al acceso a los diferentes espacios donde se desarrollan estas actividades, además de la ausencia o escasos dispositivos de asistencia, por otra parte en cuanto al Entorno Social; la totalidad de la población de personas con Discapacidad dan a conocer el buen trato que recibe por parte de sus familiares, además de los motivadores o la unión familiar existente; generando perspectivas individuales de forma significativa que le permitan cumplir con ciertas expectativas en cuanto al desempeño de determinados roles y rutinas sociales, para finalizar referente al Entorno Cultural; se muestra que en cada una de las familias caracterizadas del Municipio de Pamplona/Norte de Santander, los hogares se muestran propicios para el desarrollo y la construcción de valores, teniendo en cuenta las costumbres, creencias, patrones de actividades y comportamientos que son inculcados para de este modo facilitar el proceso de adaptación a los diferentes contextos.

De acuerdo a la variedad de patologías encontradas en el Municipio, se observó la necesidad de clasificar cada una de ellas según el tipo de Discapacidad de acuerdo a la OMS, localizando mayor énfasis en Cognitiva (25\%); Retraso Mental, Síndrome de Down, Hidrocefalia, Microcefalia, Déficit Cognitivo, Problemas de Aprendizaje y Meningitis Bacteriana, conllevando a disfuncionalidades en sus procesos de raciocinio en juicio, toma y resolución de problemas, iniciación/terminación de la actividad, seguimiento de indicaciones, aprendizaje y generalización, seguidamente Discapacidad Múltiple; Retraso en el Desarrollo Psicomotor, Distrofia Muscular, Polineuropatía, Artrogriposis, Síndrome de West y Epilépticos, por otro lado se encuentra la clasificación de Discapacidad Física, enmarcando detenidamente las siguientes Discapacidades; Parálisis Cerebral, Escoliosis, Síndrome de Raynaud y Cuadriparesia Espástica, posteriormente Discapacidades sensoriales, que incluye; Ceguera e Hipoacusia Bilateral Profunda y por ultimo Discapacidad psíquica como: 
Trastorno Mental Leve, Esquizofrenia no Especificada. Ocasionando disfuncionalidad en el desempeño de las actividades de la vida diaria (movilidad funcional, autocuidado), además las actividades básicas cotidianas, comunicación e interacción, roles; como el familiar, escolar, social, dificultando la participación social de las personas con Discapacidad por las limitaciones presentes en sus habilidades Neuromusculares, sensoriomotoras, psicosociales, requiriendo de una dependencia permanente del cuidador para suplir las necesidades y por ende mejorar la calidad de vida de los mismos.

Según resultados, la cantidad de personas que requieren de ayudas técnicas (silla de ruedas, coche, etc.), dentro de los 11 sectores localizados en el Municipio de Pamplona/Norte de Santander, son 17 personas con Discapacidad de las 60 caracterizadas. Debido a la problemática existente se observa dependencia en la ejecución de actividades diarias, restricción en la movilidad funcional, limitación de la participación en los diferentes contextos y el desempeño de los diferentes roles adquiridos dentro de la sociedad.

Dentro del Instrumento de Valoración Inicial se describe un ítem, donde se indaga acerca de los objetivos y las expectativas de los padres o cuidadores frente a la intervención desde Terapia Ocupacional, un porcentaje equivalente al $36 \%$ de los encuestados, dando respuestas en forma reiterativa que los procesos deberían ir enfocados hacia actividades de promoción de la salud, encaminadas hacia al manejo o abordaje de la patología, seguidamente con un $18 \%$ enfocados a rehabilitación en actividades de la vida diaria, por otra parte el $4 \%$ en tareas instrumentales y por ultimo ocio y tiempo libre. Referente al segundo objetivo específico del proyecto era identificar los procesos de Rehabilitación Basada en Comunidad y la Participación Social de las personas con Discapacidad según la Clasificación Internacional del Funcionamiento de la Discapacidad y de la Salud, a través de la aplicación del instrumento de valoración inicial, se logra determinar que existe un grado de dificultad moderada de las funciones corporales, a nivel de funciones mentales, sensoriales, de los sistemas digestivos, metabólicos, endocrinos y neuromusculoesqueléticas relacionadas con el movimiento.

\section{RefERENCIAS BiBLIOGRÁFICAS}

BLANCO, G, RODRIGUEZ, V. (2012) Cambios Sociales y Terapia Ocupacional. Rol del Terapeuta Ocupacional en el contexto contemporáneo. TOG (A CORUÑA) vol. 9, editorial Monog, P 190 - 205 Disponible en: http://www.revistatog.com/mono/num5/contemporaneo.pdf

BUITRAGO, M. T. (2008) Articulo producto de la investigación para optar el título de la Maestría en Desarrollo Educativo y Social. Bogotá/Colombia.

CRUZ, I. DUARTE, C. FERNÁNDEZ, A.GARCíA, S. (2010) Secretaría Distrital de Salud, Hospital Centro Oriente - Universidad del Rosario "Fortalecimiento de la Red de Rehabilitación Basada en Comunidad de la Secretaría Distrital de Salud". 2005-2010. Bogota.

DHUNAGNA, BM. (2003) Las vidas de mujeres con discapacidad en Nepal: Vulnerabilidad sin apoyo. Master's thesis, Thailand, Instituto Asiático de tecnología. Editorial Pnuma, P 25.

FRANSEN, H. (2002) El dominio de las ocupaciones de la vida diaria: La experiencia y el sentido en dos familias de niños con necesidades especiales. Amsterdam: Instituto de Terapia Ocupacional. Editorial Board

GUÍA PARA PADRES DE NIÑOS Y NIÑAS CON PARÁLISIS CEREBRAL Y RETRASO MOTOR. ( 1988) Elaborado por Equipo Terapéutico del Centro de limitados Neuromusculares y Ortopédicos, Secretaria de Educación - Sección de Educación Especial - Cúcuta, Primera Edición.

ORGANIZACIÓN MUNDIAL DE LA SALUD. (2012) Guía para la rehabilitación basada en la comunidad. Editorial Panamericana,

MABANGWE, J.P. \$(2010) Discapacidad para convertirse en madre o padre y disfrutar los beneficios en la comunidad, Rehabilitación de base comunitaria, red de África. Editorial Cinca, P 66 - 196

MONGUÍ, M. M. (2009) Asociación de Discapacidad de Piedecuesta: ASODISPIE. Santander - Colombia.

ORGANIZACIÓN MUNDIAL DE LA SALUD. (2012). Guía para la Rehabilitación Basada en la Comunidad, según Lord JE, Guernsey KN, Balfe JM, Karr VL, Flowers N (eds), 2007, ¡Derechos humanos sí! Acción y defensa de los derechos humanos de las personas con discapacidad], Universidad de MinnesotaCentro de recursos de derechos humanos, Minnesota.

ORGANIZACIÓN MUNDIAL DE SALUD. (2003) Organizaciones de las Naciones Unidas, Organizaciones no Gubernamentales (ONGs), Organizaciones de Personas con Discapacidad (OPDs) y el Ministerio de asuntos sociales y salud. Helsinki, Finlandia.

UNIVERSIDAD NACIONAL DE COLOMBIA Editora Ana Doris Cifuentes - Sara Ximena Rubio y Jeannette Amanda Méndez Montaño. (2007) Estrategias de acompañamiento a procesos de organización de hombres y mujeres en situación de Discapacidad. Instituto de Desarrollo Humano y Discapacidad, Ocupación Humana y Movimiento Corporal Humano, Bogotá, D.C - Colombia. Editorial Guadalupe. 


\section{ANEXOS}

\section{VISITA FALLIDA}

\begin{tabular}{|c|}
\hline Localidad: Hospital: \\
\hline Nombre: Edad: \\
\hline UPZ/Barrio: Fecha: \\
\hline Hora: \\
\hline OBSERVACIONES: \\
\hline
\end{tabular}

Firma del Profesional:

Firma de un Testigo:

\section{INSTRUMENTO DE EVALUACION INICIAL}

\begin{tabular}{|c|c|}
\hline Localidad: & Hospital: \\
\hline Barrio & UPZ: \\
\hline $\begin{array}{l}\text { Nombre Del Usuario } \\
\text { D.I: }\end{array}$ & $\begin{array}{l}\text { Fecha de Nacimiento: } \\
\text { Día_/Mes_/Año__ } \\
\text { Edad: Sexo: F__ }\end{array}$ \\
\hline Dirección Residencia: & Teléfono \\
\hline Escolaridad: & Ocupación: \\
\hline $\begin{array}{l}\text { Nombre del Cuidador } \\
\text { Edad: }\end{array}$ & Parentesco \\
\hline \multicolumn{2}{|c|}{ Rehabilitación Funcional Si___ No ___ Institución } \\
\hline $\begin{array}{l}\text { Seguridad Social } \\
\text { EPS/ARS/ARP/Otro }\end{array}$ & IPS \\
\hline \multicolumn{2}{|l|}{ Fecha de la Visita } \\
\hline Profesional Responsable de la Asesoría & Profesión: \\
\hline
\end{tabular}


DIAGNOSTICO (CIE 10)

LIMITACIONES PERMANENTES (CIF):

REQUERIMIENTO DE AYUDAS TÉCNICAS: (especifique motivos)

GENOGRAFA, FAMILIOGRAMA, COMPOSICION FAMILIAR

MAPA DE REDES DE

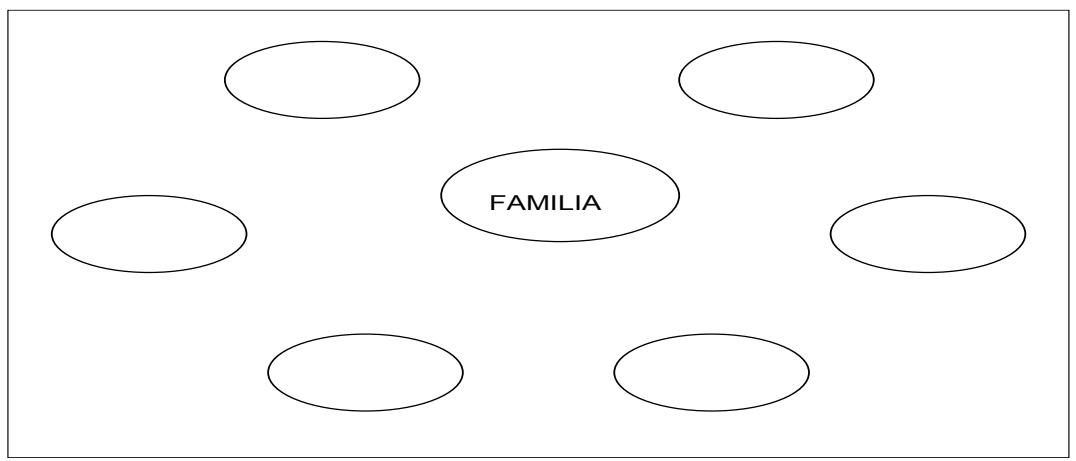

\section{OBSERVACIONES:}

NARRATIVA: (versión de la familia en relación con los hallazgos de la evaluación y aplicación del instrumento, es importante enfatizar en las barreras y facilitadores encontrados) (no debe pasar de dos cuartillas)

OBJETIVOS Y ESPECTATIVAS DE LA FAMILIA:

Firma de la persona con discapacidad o cuidador

Firma del profesional 


\section{LISTA DE CHEQUEO}

\section{OBJETIVO DE LA INVESTIGACIÓN:}

Determinar el nivel de incidencia de la participación social, a través de intervenciones asistenciales en el proceso de Rehabilitación Basada en Comunidad en las personas con discapacidad en el municipio de Pamplona - Norte Santander

\section{LISTA CHEQUEO:}

Lea atentamente las preguntas y Marque con una " $X$ " su respuesta según corresponda.

\begin{tabular}{|c|c|c|}
\hline ENTORNOS & \multicolumn{2}{|c|}{ CALIFICACIÓN } \\
\hline SOCIAL & PRESENTE & AUSENTE \\
\hline & & \\
\hline & & \\
\hline & & \\
\hline & & \\
\hline & & \\
\hline FÍSICO & & \\
\hline & & \\
\hline & & \\
\hline & & \\
\hline & & \\
\hline & & \\
\hline & & \\
\hline & & \\
\hline & & \\
\hline & & \\
\hline
\end{tabular}

